\title{
Protection de la jeunesse sans interdiction de la publicité?
}

\section{Rainer M. Kaelin}

Dr méd., spécialiste en Médecine interne et Pneumologie, membre FMH

La Loi sur les produits du tabac (LPTab) fut renvoyée au Conseil fédéral avec le mandat d'y ancrer la protection de la jeunesse, mais de supprimer les restrictions de la publicité. Les votes du parlement [1]* laissent perplexe. Le conseiller des Etats Dittli (PLR) déclara ne pas connaître des données "qui prouvent que les quotas de fumeurs diminuent à cause des interdictions de la publicité» et sa collègue, Mme Keller-Suter (PLR), ne comprend pas: «Ceci n'est pas justifié plus en détail, on établit uniquement une corrélation.» Bien que l'interdiction globale de toute publicité, promotion et parrainage pour le tabac conditionne la ratification de la convention cadre de l'OMS pour combattre l'épidémie du tabac, ce traité ne fut mentionné par aucun parlementaire de la majorité.

Cet article décrit «l'autocontrôle» de la publicité pour le tabac et pourquoi une protection de la jeunesse sans interdiction globale de la publicité n'est pas possible.

\section{La commission de "Loyauté»}

L'Association suisse des fabricants de cigarettes (ASFC) qui précéda Swiss Cigarette indique dans son code d'éthique de 1966 [2] le public cible: «Puisque la jeunesse est influençable par nature, l'ASFC soumet la publicité à des restrictions.» La publicité visant les

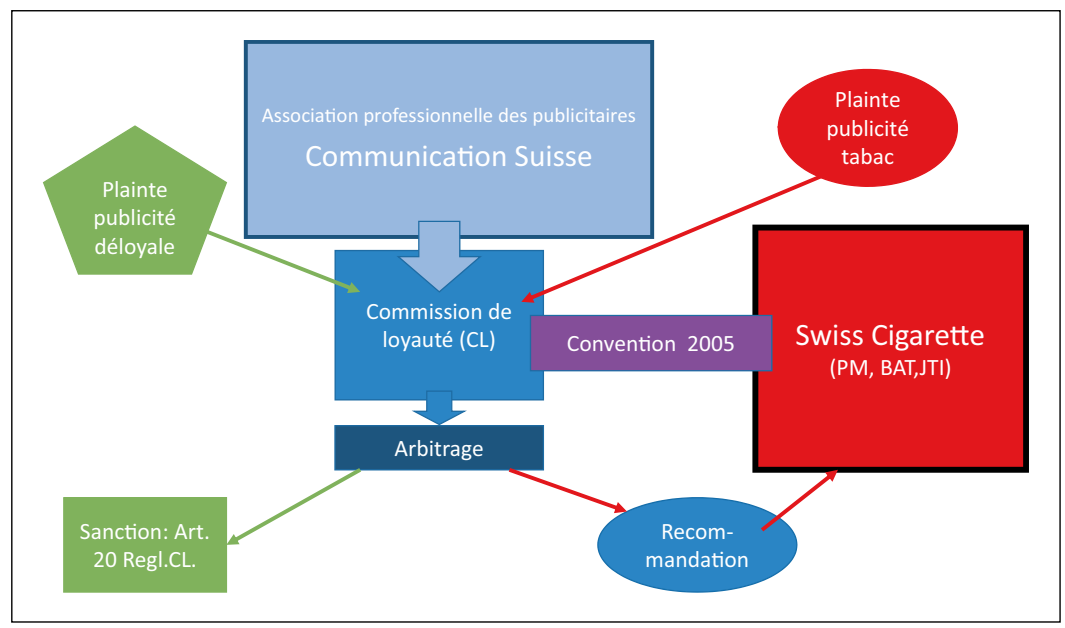

Figure 1: Commission de loyauté, diagramme fonctionnel. PM = Philip Morris; BAT = British American Tobacco; JTI = Japan Tobacco International jeunes de moins de 18 ans, montrer des personnes qui visiblement ne sont pas adultes, des stars de cinéma et des chanteurs sont interdits. La convention [3] entre Swiss Cigarette et la commission de loyauté de l'association des publicitaires, signée en 2005 par sa présidente Doris Leuthard, confirme l'interdiction de la publicité ciblant spécifiquement les jeunes. Celle-ci est définie comme "information d'un fabricant pour le choix d'une marque parmi d'autres». La publicité «ne doit pas présenter de célébrités [...] et ne doit pas lui faire porter une message implicite ou explicite [...], représenter une personne de moins de 25 ans, suggérer que fumer favorise le succès sportif, social, professionnel, ou sur le plan sexuel». Les limites laissent place à l'interpretation: «... sauf si on peut présumer qu'au moins $80 \%$ des lecteurs sont adultes»; "... excepté (qu')au moins 75\% du public est adulte»; «sauf si le fabricant s'assure que la personne demandant accès (à l'internet) prouve qu'elle est adulte»; "sauf si elles sont sponsorisées par un fabricant», mais alors celui-ci doit s'assurer que les manifestations promotionnelles sont «uniquement accessibles aux adultes». La commission de loyauté seule est compétente pour «constater des infractions». «La recommandation définitive est portée à la connaissance de Swiss Cigarette. Dans le cas d'une infraction [...] cette dernière se charge de la porter devant l'arbitrage interne. L'article 20 de la commission de loyauté n'est pas applicable» [4]. Ce fonctionnement est présenté dans la figure 1, montrant que la commission ne sanctionne pas les infractions, mais les transmet comme recommandations à Swiss Cigarette.

\section{Signification de «l'autocontrôle de la publicité»}

Puisqu'une moitié de ses clients meurt, l'autre devient malade, et la majorité des fumeurs cherche à arrêter, la publicité est une question de survie pour l'industrie. Avec deux fonctions: pour les fumeurs dépendants tout élément rappelant la fumée déclenche le réflexe d'allumer une cigarette. Secundo, la publicité amène des nouveaux clients vers la nicotine. Car malgré la toxicité notoire du tabac, la publicité doit rendre plau- 
sible que ses produits sont des bien de consommation légaux, comme les vêtements ou les parfums, comparables à d'autres marques; ils doivent apparaître comme des éléments normaux de notre société de consommation. Une jeune personne choisit avec une marque de cigarettes son "style» et s'identifie avec ses valeurs, comme le prestige, le glamour, etc. Que la publicité serve uniquement à informer les consommateurs des qualités du produit, dans le cas d'un «bien» qui rend dépendant, est une tromperie. Car les rares fumeurs adultes changeant de marque ne justifient jamais les sommes investies dans leur promotion [5, 6]. L'importance primordiale de viser la jeunesse provient du fait que l'addiction à la nicotine (comme celle de l'héroine, de l'alcool, du cannabis, etc.) se fixe plus facilement dans le cerveau en développement; les personnes devenues dépendantes jeunes ne peuvent se libérer que difficilement. Des documents internes indiquent, ce que les données sur les jeunes fumeurs démontrent, c.à.d. que l'industrie cache, à l'aide de "l'autocontrôle» et de la commission de "loyauté», ses intentions: "Younger adult smokers are the only source of replacement smokers [...]. If younger adult smokers turn away from smoking, the industry must decline, just as a population which does not give birth, will eventually dwindle» [7]. La conclusion de la Federal Trade Commission en 1967 reste d'actualité: «Self regulation by the industry has proved to be ineffectual [...]. Today teenagers are constantly exposed to subtle messages that cigarette smoking increases popularity, makes one more masculine, etc. To allow [...] especially teenagers the opportunity to make an informed and deliberate choice of whether or not to start smoking, they must be freed from constant exposure [...]" [8].

\section{Manipulation psychologique}

«La première cigarette pour un débutant est une expérience déplaisante. Pour expliquer qu'il tolère cet aspect désagréable, il nous faut invoquer un motif psychosocial. Fumer [...] est un acte symbolique. Le fumeur déclare à son entourage: 'voilà le type de personne que je suis'. Lors que la force du symbole psychologique faiblit, c'est l'effet pharmacologique qui prend la relève» [9]. «En plus, à cet âge tombe le choix de la marque [...]» [10]. Clotaire Rapaille [11] disséqua en 1991 dans le projet Archetype pour Philip Morris (PM) les motifs des jeunes [12]. Il articule le processus en stade I, «impression/prise de conscience», et stade II, "rite de transition». Dans le stade I, l'adolescent devient conscient que fumer est une activité plaisante, réservée aux adultes, dont il est exclu. Des associations se forment d'appartenance/exclusion du groupe, et entre fumer / être adulte. Dans le stade II, il brise le tabou en allumant la cigarette et joint le groupe des fumeurs/adultes. Basé sur cette analyse, Rapaille recommande la stratégie [12]: "Stress that smoking is for adults only; Make it difficult for minors to obtain cigarettes; Continue to have smoking perceived as a legitimate, albeit morally ambiguous adult activity [...] between activities that everyone can partake in and activities that only the fringe of the society embraces; Stress that smoking is for people who like to take risks, who are not afraid of taboos, who take life as an adventure to prove themselves."

Avec «MayBe», PM réalisa en 2012 une promotion par presse gratuite et Internet. Elle montra un jeune qui répond par «may be» aux messages du type: «La vie est là pour être vécue, pour se faire plaisir. Le 'peut-être' laisse filer une occasion.» L'invitation au Marlboro-event déclare: "A MayBe is not invited. Don't be a MayBe, be Marlboro.» On distingue exclusion/plaisir, invitation/ risque, briser le tabou. Cet action a été dénoncée comme infraction du code à la commission de la loyauté, qui refusa la plainte [13]

\section{«Communication» par multiples canaux}

La publicité classique, jamais «neutre», est reconnaissable et peut être relativisée. Dans la communication commerciale cependant, la publicité identifiable en tant que telle est uniquement une partie de la manipulation. Le "content marketing" touche les groupes cible indirectement. Des «journalistes d'entreprise» planifient avec des agences de relations publiques des contenus rédactionnels [14] sans mention du produit ni du fabricant, et diffusent ceux-ci parmi des experts, fonctionnaires, politiciens, secrétaires d'associations, etc. Ces "formateurs de l'opinion" font passer le message autour d' eux et rendent l'environnement réceptif à la publicité directe [15]. Le succès du content marketing est inversement proportionnel à sa transparence. Les relais des messages ne sont guère conscients qu'ils sont instrumentalisés. Manque d'argent des médias, journaux gratuits, périodiques et newsletters d'associations et d'organisations de santé, etc., ainsi que l'Internet favorisent la technique. Ceci incita récemment le Conseil de la presse à postuler plus de transparence entre information indépendante et celle téléguidée par des relations publiques [16].

\section{L'e-cigarette "prouvée moins nocive»}

Pour l'industrie, l'e-cigarette (ainsi que les «heat but not burn devices») est un instrument de marketing. Par son «innocuité», elle est proposée comme un jouet banal auprès des enfants, dont les parents interdisent de 
Tableau 1: Diminution annuelle en pourcent du nombre de fumeurs 1990-2015 et 2005-2015, Suisse et pays voisins; ainsi que USA/Australie, et Europe du Nord.

\begin{tabular}{lllll}
\hline & $\mathbf{1 9 9 0 - 2 0 1 5}$ & & 2005-2015 & \\
\hline Australie & Hommes & Femmes & Hommes & Femmes \\
\hline USA & $-2,1$ & $-1,9$ & $-1,9$ & $-2,2$ \\
\hline Autriche & $-2,2$ & $-2,0$ & $-2,0$ & $-2,4$ \\
\hline France & $+0,3$ & $-0,3$ & $-0,8$ & $-0,2$ \\
\hline Allemagne & $-0,6$ & $-1,5$ & $-1,0$ & $-1,6$ \\
\hline Italie & $-0,3$ & $-0,9$ & $-0,5$ & $-0,6$ \\
\hline Suisse & $-0,9$ & $-1,1$ & $-1,0$ & $-0,9$ \\
\hline Suède & $-1,4$ & $-1,3$ & $-1,8$ & $-1,6$ \\
\hline Norvège & $-2,7$ & $-3,2$ & $-3,1$ & $-1,4$ \\
\hline Danmarque & $-2,6$ & $-2,8$ & $-2,4$ & $-3,1$ \\
\hline & $-3,0$ & $-3,0$ & $-2,3$ & $-3,8$
\end{tabular}

fumer. Avec «réduction du risque», on en fait la promotion en tant qu'aide à l'arrêt du tabagisme ou remplacement de la cigarette tabac. Les arguments de promotion pour enfants et adultes sont presque identiques. La différence réside uniquement dans les liquides contenant ou pas de la nicotine. Dans le stade I selon la stratégie de PM, les e-cigarettes et e-shishas conduisent les enfants vers l'adolescence en les excluant de nicotine et de la fumée. Dans le stade II, l'e-cigarette fait appel à la prise de risque des jeunes; le tabou est brisée par la première cigarette tabac par laquelle ils joignent le groupe des adultes/fumeurs. Avant, ils expérimentent avec les arômes tabac et les liquides nicotinés. Ainsi, ils vivent la première cigarette tabac d'autant plus agréablement, qu'elle livre la nicotine plus rapidement à leur cerveau déjà sensibilisé. Cette stratégie correspond aux investissements de l'industrie dans l'e-cigarette [17] et elle se confirme par les données épidémiologiques. L'e-cigarette est devenue un problème chez les NordAméricains de 18 à 24 ans [18] par le passage à la cigarette tabac bien documenté par plusieurs études [19-21]. La notion de sa "nocivité diminuée de $95 \%$ » se base probablement sur du content marketing. Car cette affirmation ne provient d'aucune donnée mesurée, mais uniquement d'avis subjectifs prononcé en 2013 par un

Les documents internes de l'industrie de tabac et la description de la stratégie PM de C. Rapaille m'ont été mis à disposition par Pascal Diethelm, président d'OxyRomandie que je remercie ici chaleureusement. II les a présentés en conférence devant le $10^{\mathrm{e}}$ congrès de la Société Francophone de Tabacologie à Lille, France, le 2.11.2016. groupe de consultants qui recommandent de préférence l'arrêt du tabagisme par l'e-cigarette [22].

\section{Conclusions}

De son modèle d'affaire découle que l'industrie vise la jeunesse pour survivre. Car elle ne peut guère convaincre d'emblée les adultes à acheter son produit toxique qui rend dépendant. Elle se sert de l'e-cigarette pour conditionner les enfants au comportement de fumeurs et pour les habituer à la nicotine. L'autocontrôle de la publicité pour la protection de la jeunesse ne peut remplir sa mission, car ce système n'est pas indépendant, les infractions restent sans sanctions, et les messages proférés «à l'adresse des adultes uniquement» touchent justement spécialement les jeunes.

Au vu de la publicité du tabac basée sur la psychologie de l'adolescence, il convient de repenser les actions préventives ciblant les jeunes. Car si celles-ci suggèrent entre les lignes que fumer est discutable et que le tabac doit rester réservé au libre choix d'adultes, elles aident la publicité du tabac par le goût du risque et de la transgression qui conduit les jeunes par leur nature à briser le tabou au moyen de la cigarette tabac. Pour cette raison, l'interdiction de vente aux mineurs dans la LPTab (point sur lequel les parlementaires et les cigarettiers sont d'accord) sera inefficace sans l'interdiction globale de la publicité.

Le content marketing sous-liminal de l'industrie invite le parlement de réaliser la protection de la jeunesse dans la LPTab par une approche pragmatique et basée sur les faits. Celle-ci n'est pas une question de gauche ou de droite, mais si oui ou non la santé des jeunes est à considérer une valeur supérieure au gain financier particulier. Les formules idéologiques du type "pour ce qui peut être acheté légalement, on doit pouvoir faire de la publicité» [23] ne sont pas utiles à l'égard de produits dont les fabricants déjouent la prévention depuis des décades. Des mesures cantonales sont également futiles. En comparant la diminution annuelle du nombre de fumeurs dans divers pays, il est évident (tab. 1) que les réglementations globales selon l'OMS des pays nordiques s'avèrent efficaces [24].

Crédit illustration

Graphique R. M. Kaelin 